\title{
Survey of flexible fibreoptic bronchoscopy in the United Kingdom
}

\author{
C.M. Smyth, R.J. Stead
}

\begin{abstract}
Survey of flexible fibreoptic bronchoscopy in the United Kingdom. C.M. Smyth, R. J. Stead. (C) ERS Journals Ltd 2002.

ABSTRACT: The practice of flexible bronchoscopy is not standardized. Current guidelines are concerned primarily with safety aspects of the procedure. In view of this, and the authors' own observations of individual variation in preparation and technique, a national survey of bronchoscopic procedure was performed to assess physicians'
\end{abstract} methods.

A structured questionnaire was mailed to 547 consultant physicians in adult respiratory medicine. Physicians' routines of patient preparation, drug therapy, sampling methods, and experience of complications with the flexible bronchoscope were assessed.

A $60 \%$ response (328 physicians) was obtained. Patient consent was obtained by a junior doctor in $31 \%$ of replies. $205(63 \%)$ physicians gave benzodiazepine sedation, 46 (14\%) used opioid, and $38(12 \%)$ administered both. Ninety-four $(29 \%)$ physicians prescribed an antimuscarinic agent, and $235(74 \%)$ gave antibiotics to patients with mechanical heart valves. Only $22 \%$ of physicians monitored electrocardiogram and $10 \%$ monitored blood pressure during all procedures. Marked variance was noted in sampling routines of suspected lung tumours. Physicians who used fluoroscopic guidance for transbronchial lung biopsy reported a significantly lower incidence of pneumothorax requiring drain insertion over the previous 12 months compared to those who did not $(2.68$ of 1000 versus 9.17 of $1000,(p<0.03))$, but no difference in the total incidence of pneumothorax. Only $87(27 \%)$ of responders had performed transbronchial needle aspiration sampling over the previous 12 months.

The preparation and practice of flexible bronchoscopy varies greatly for each physician. Use of radiographical screening for performing transbronchial lung biopsy was associated with a lower likelihood of pneumothorax requiring chest tube drainage. Eur Respir J 2002; 19: 458-463.
Macclesfield District General Hospital, Victoria Road, Macclesfield, Cheshire, SK10 3BL, UK.

\author{
Correspondence: C.M. Smyth \\ 5 Ryder Crescent \\ Aughton \\ Ormskirk \\ Lancashire \\ UK \\ L39 5EY \\ Fax: 441625663150
}

E-mail: colin.smyth@tesco.net

Keywords: Flexible fibreoptic

bronchoscopy

national survey

United Kingdom

Received: December 122000

Accepted after revision September 14 2001

This study was funded by the East Cheshire NHS trust.
It is now $>30 \mathrm{yrs}$ since IKEDA [1] introduced the technique of flexible fibreoptic bronchoscopy, and this procedure has been established throughout the UK for $>20$ yrs. Despite this, bronchoscopic practice in UK hospitals is not standardized. National guidelines on flexible bronchoscopy concentrate predominantly on safety aspects of the procedure, rather than practical aspects of the technique [2]. In view of this, the authors undertook a national survey of flexible bronchoscopy which focused primarily on physicians' preferences for patient preparation, monitoring during the procedure, drug therapy and tissue sampling methods. Information concerning physicians' experiences of complications and of performing therapeutic procedures with the flexible bronchoscope was also sought. The aims of the study were to assess the extent of variation in physicians' common practice, to compare activity with evidence-based data, and to assess the degree of adherence to accepted standards of practice.

\section{Method}

Consultant physicians in adult respiratory medicine in the UK were identified by the national directory of the British Thoracic Society. A structured questionnaire was sent by post to the hospital addresses of all doctors listed, although it was anticipated that not all physicians contacted performed bronchoscopy. The questionnaire dealt with several areas of practice, including: 1) patient preparation and investigations prior to bronchoscopy, duration of fasting prior to bronchoscopy and patient consent; 2) drug administration for bronchoscopy, including methods of sedation, topical anaesthesia, and prophylactic antibiotic therapy; 3) patient monitoring during the procedure; 4) endoscopist clothing/protection; 5) sampling methods via the bronchoscope; 6) experience of therapeutic procedures performed via the bronchoscope; 7) experience of complications/death.

Responders were asked to provide detailed comment on all areas covered. To encourage a high response rate, replies were anonymous and nontraceable. Neither second questionnaires nor reminders were sent. Data were obtained only from physicians' practicing in statefunded hospitals; thus there was no financial incentive to perform bronchoscopy. Comparisons were made using Fisher's exact test, where appropriate.

\section{Results}

A total of 547 questionnaires were sent to consultant respiratory physicians. Three-hundred and 
twenty-eight were returned, of which 27 were incompletely answered. A further 15 physicians replied, but did not perform bronchoscopy; thus an overall response rate of $62.7 \%$ was attained. Over the 12 -month period prior to answering the questionnaire, approximately 60,100 bronchoscopic examinations had been performed or supervised by the 328 responders (median 175 procedures $\cdot$ physician $^{-1} \cdot \mathrm{yr}^{-1}$ (range: 5-1000)) (table 1).

\section{Patient preparation}

A total $8.4 \%$ of physicians routinely fasted patients for $>8 \mathrm{~h}$ before bronchoscopy, $77.3 \%$ fasted patients for between $4-8 \mathrm{~h}$ and $14.3 \%$ for $<4 \mathrm{~h}$. In the latter group of 46 physicians, 41 used sedation for bronchoscopy. Informed patient consent was obtained by either a middle or consultant grade physician in $66 \%$ of replies, by a junior doctor in $31 \%$, and by nursing staff in $2 \%$.

Spirometry was arranged by two-thirds of physicians prior to bronchoscopy. Two physicians routinely obtained baseline arterial blood gases (ABG), and a further 206 of $326(63 \%)$ physicians obtained ABG before bronchoscopy if poor oxygenation was suggested by clinical status, low oxygenation as measured by pulse oximeter, or poor spirometric performance.

Prior to bronchoscopy without transbronchial biopsy (TBB), a full blood count (FBC) was requested routinely by $57 \%$ of physicians, blood urea and electrolytes by $43 \%$, and clotting function by $18 \%$. Before bronchoscopy with TBB, $91 \%$ of responders requested $\mathrm{FBC}, 88 \%$ requested tests of clotting function, $10 \%$ had blood grouped and saved and $73 \%$ obtained spirometry or ABG.

\section{Drug therapy for bronchoscopy}

Sedation regimes are shown in table 2. All but one physician who used opioid or benzodiazepine reported having ready access to the appropriate pharmacological antagonist in the bronchoscopy room. Several physicians commented that they administered sedation as they felt it improved tolerance of the procedure, whilst others gave sedation only to patients who were particularly anxious. Topical lidocaine was the most commonly used local anaesthesic. A total of 193 of the 280 endoscopists who passed the bronchoscope via the nose, applied lidocaine gel intranasally, 62 physicians used lidocaine spray, and five used

Table 1. - Number of flexible bronchoscopic examinations performed by responding physicians over a previous 12-month period

\begin{tabular}{cr}
\hline Bronchoscopies $\cdot \mathrm{yr}^{-1} \mathrm{n}$ & Physicians $\mathrm{n}(\%)$ \\
\hline$<100$ & $38(12)$ \\
$100-199$ & $133(42)$ \\
$200-299$ & $107(34)$ \\
$>299$ & $37(12)$ \\
\hline
\end{tabular}

Table 2.-Physicians' preferred sedation regimen for flexible bronchoscopy

\begin{tabular}{lc}
\hline Sedation regime & Physicians \\
\hline Benzodiazepine alone & $207(63)$ \\
Opioid alone & $45(14)$ \\
Benzodiazepine and Opioid & $38(12)$ \\
Other & $3(1)$ \\
No sedation & $34(10)$ \\
\hline
\end{tabular}

Data are presented as n (\%).

topical cocaine as the main form of nasal anaesthesia. Twenty physicians reported no preferred method of anaesthesia.

Forty-eight $(15 \%)$ responders routinely administered local anaesthetic by transtracheal injection (lidocaine in 32 cases, cocaine in 16). Eighteen physicians prescribed lidocaine via nebulizer. Lidocaine was given in a "spray as you go" fashion to the vocal cords and beyond via the bronchoscope by $266(81 \%)$ of responders. Physicians were asked to quantify the usual total amount of lidocaine administered during bronchoscopy and this was stated in 270 replies. Assuming doses of lidocaine gel and spray of $100 \mathrm{mg}$ and $60 \mathrm{mg}$, respectively were applied to the upper airway, the median total dose was $380 \mathrm{mg}$ (range: 80-960). It was estimated that a total dose of lidocaine in excess of $5 \mathrm{mg} \cdot \mathrm{kg}$ body weight ${ }^{-1}$ was given by $53 \%$ of physicians.

Ninety-four (29\%) endoscopists gave an antimuscarinic agent. One-hundred and ninety-three $(60.5 \%)$ physicians prescribed antibiotic prophylaxis to patients undergoing bronchoscopy who had known heart-valve defects. A further 13\% administered antibiotics only to patients deemed at high risk of developing endocarditis (previous endocarditis or mechanical heart valves).

\section{Monitoring and support during bronchoscopy}

Patient monitoring and support during the procedure are outlined in table 3 . Seven responders who routinely gave intravenous sedation did not maintain venous access during bronchoscopy. Blood pressure was taken by most responders prior to bronchoscopy, rather than during the procedure. The numbers of trained assistants present during bronchoscopy varied between one and six, with 19 physicians reporting routine attendance of a single assistant only.

Table 3.-Frequency of use of monitoring and support during bronchoscopy

\begin{tabular}{lccc}
\hline & Always & Sometimes & Never \\
\hline Pulse oximeter & 99 & 1 & 0 \\
ECG monitor & 22 & 24 & 54 \\
Supplemental oxygen & 66 & 34 & 0 \\
Venous cannula & 87 & 8 & 5 \\
BP & 10 & 8 & 82 \\
\hline
\end{tabular}

Data are presented as \%. ECG: electrocardiogram; BP: blood pressure. 
Table 4.-Frequency of use of protective clothing when bronchoscoping patients at high risk and low risk for transmission of infectious disease

\begin{tabular}{lcccc}
\hline & Gloves & Gown & $\begin{array}{c}\text { Eye } \\
\text { protection }\end{array}$ & $\begin{array}{c}\text { Facial } \\
\text { mask }\end{array}$ \\
\hline Low risk & 98 & 62 & 16 & 25 \\
High risk & 100 & 91 & 73 & 90 \\
\hline
\end{tabular}

Data are presented as $\%$.

\section{Endoscopist clothing and protection}

Details of protective equipment worn by physicians are shown in table 4 . Only $29(9 \%)$ responders wore all four items (gloves, gown, eye protection and face mask) routinely for all procedures, and 204 (63\%) wore all items whilst bronchoscoping patients felt to be high risk for transmission of infective agents.

\section{Sampling techniques}

Physicians were asked about their preferences for bronchoscopic sampling methods for patients with suspected bronchial carcinoma and mass lesion as seen on the chest radiograph. Separate answers were obtained for three possible types of bronchoscopic appearance: normal $(\mathrm{N})$, visible occluding tumour (VOT), and mucosal swelling/irregularity (MSI). The frequency of the physicians' usual sampling regimes using endobronchial biopsy, brush biopsy and bronchial washings for histocytological analysis when presented with the three appearances is shown in table 5. In addition to these samples, transbronchial needle aspiration (TBNA) was occasionally performed by 10,3 and $9 \%$ of physicians when obtaining samples with appearances N, VOT and MSI, respectively.

Information concerning physicians' experience of TBB was obtained. Of 308 responders, 109 (35\%) routinely used fluoroscopic guidance when performing TBB. One-hundred and seventy-five physicians (57\%) never used fluoroscopy for TBB, whilst 24 (8\%) reported its occasional use. The number of TBBs performed by physicians over the previous 12 months

Table 5.-Physicians' usual policy of histocytological sampling via the bronchoscope from patients with a radiographical mass lesion

\begin{tabular}{lccc}
\hline Sampling regime & \multicolumn{3}{c}{ Bronchoscopic appearances } \\
\cline { 2 - 4 } & Normal & $\begin{array}{c}\text { Visible } \\
\text { tumour }\end{array}$ & $\begin{array}{c}\text { Irregular/narrow } \\
\text { mucosa }\end{array}$ \\
\hline BI alone & 0 & 6.4 & 2.5 \\
BI and W alone & 3.7 & 18.4 & 7.7 \\
BI and BR alone & 0 & 15.1 & 10.5 \\
BI, W and BR & 11.7 & 59.8 & 78.7 \\
W alone & 26.5 & 0 & 0 \\
BR alone & 3.1 & 0 & 0 \\
BR and W alone & 50.9 & 0.3 & 0.6 \\
No samples & 4.1 & 0 & 0 \\
\hline
\end{tabular}

Data are presented as \%. BI: biopsy; W: wash; BR: brush. varied greatly (0-250 (median 8.0)). When comparing physicians who always used fluoroscopic guidance for TBB with those who never used it, no difference was noted in the reported total frequency of pneumothorax in the previous 12 months (8.57 of 1,000 versus 11.46 of 1,000 , respectively), although there was a significant difference in the reported rate of pneumothorax requiring tube drainage $(2.68$ of 1,000 with fluoroscopy versus 9.17 of 1,000 without fluoroscopy; $\mathrm{p}<0.03$ ). A plain chest radiograph post-TBB was requested by a higher proportion of those who used fluoroscopy than those who did not, $(92 \%$ versus $81 \%$, respectively). Forty-six (15\%) physicians routinely admitted patients overnight after performing TBB.

Experience of TBNA in the previous 12 months was limited to $85(27 \%)$ physicians, with a total of 452 procedures having been performed. Only six physicians used radiographical guidance when performing TBNA. There was one reported pneumothorax and no episodes of significant haemorrhage. Several physicians commented on experience of poor diagnostic yield from TBNA.

\section{Therapeutic procedures performed via the bronchoscope}

Forty-eight (15\%) physicians had performed endobronchial stenting via the flexible bronchoscope in the previous 12 months, with a total of 166 procedures being undertaken. An early complication rate of $6.6 \%$ was reported from this procedure. Thirty-six physicians reported recent experience of other specialist procedures via the flexible bronchoscope, including brachytherapy (18 physicians), laser therapy (16), phototherapy (five), balloon dilatation (three), diathermy (three), fluorescence bronchoscopy (two) and electrocoagulation (one).

\section{Mortality from bronchoscopy}

A total of 27 deaths were reported (mortality $0.045 \%$ ). Of these, 11 patients had advanced bronchial malignancy. A further three patients died from cardiac disease, and, two succumbed to pre-existing pulmonary sepsis. No details were given concerning seven further deaths. No deaths attributable to TBB were reported. There was no apparent association between deaths from bronchoscopy and any specific sedation regime, number of bronchoscopies performed per physician, or topical anaesthetic dose. Use of sedation was not associated with greater mortality than bronchoscopy without sedation.

\section{Discussion}

These findings confirm that the practice of flexible bronchoscopy varies greatly. This is likely to result from a combination of individual familiarity with learned techniques, and the presence of conflicting data regarding many aspects of bronchoscopic procedure. However, when areas of management for 
which standards of care are already established were examined, considerable variation was noted also.

Patient consent was obtained by a junior doctor in almost one-third of responses. It is likely that a proportion of these doctors did not discuss intricate details/possible complications of bronchoscopy, due to lack of familiarity with the technique. A recent review of litigation cases arising from gastrointestinal endoscopy in the UK revealed more than one-third of claims related to poor patient explanation when obtaining consent [3]. The authors believe that consent to bronchoscopy should only be obtained by personnel familiar with the procedure and its possible complications.

The authors were surprised by the number of physicians who fasted patients for $<4 \mathrm{~h}$ before bronchoscopy. When administering sedation and topical anaesthetic agents, it is probably prudent to fast patients for at least $4 \mathrm{~h}$. Prior assessment of bloodclotting function has been shown to be poorly predictive of haemorrhage in patients without risk factors for bleeding, who undergo bronchoscopy without TBB. The current finding that $18 \%$ of UK physicians routinely check blood clotting before bronchoscopy in such patients was much lower than the $44 \%$ of physicians in a recent North American survey who did likewise [4]. Assessment of spirometric performance is unlikely to influence bronchoscopy, provided continuous assessment of oxygen saturation is made. Although almost all physicians now monitor oxygen saturation routinely during flexible bronchoscopy, the proportion requesting prior pulmonary function testing is unchanged from a previous British survey in 1983 [5].

There has been debate concerning the need for sedation in flexible bronchoscopy. Sedation has been implicated as the cause of up to $50 \%$ of major complications resulting from bronchoscopy [6]. HАтTON et al. [7], in a double-blind trial, showed no improvement in patient comfort when comparing midazolam $70 \mu \mathrm{g} \cdot \mathrm{kg}^{-1}$ versus placebo. However, other studies have not confirmed these findings. Putinati et al. [8] showed significant improvement in patient tolerance when intravenous diazepam was given. This study also demonstrated a significant reduction in the number of abandoned procedures due to patient intolerance when sedation was given. The need for sedation is likely to vary among patients, and may be affected by the extent of explanation and reassurance given by medical staff. The finding that $7.3 \%$ of physicians performed bronchoscopy without sedation is similar to the $4 \%$ of physicians who did likewise in a recent American study [4]. The fact that most physicians administered sedation suggests that it is generally felt that this practice is relatively safe. The combined use of opioid with benzodiazopine, however, can be questioned as this has not been shown to improve patient tolerance compared to either agent alone and may increase the risk of cardiorespiratory depression [9, 10].

Most endoscopists used lidocaine for topical anaesthesia. Only a minority favoured intranasal lidocaine spray, which produces greater patient discomfort than lidocaine gel [11]. Lidocaine was administered via nebulizer by a small number of physicians. Despite high patient acceptability for this method [12], it may induce bronchospasm in susceptible patients [13]. A large number of physicians reported use of total lidocaine doses in excess of previously recommended amounts $\left(5 \mathrm{mg} \cdot \mathrm{kg}^{-1}\right)$. Despite this, there were no reported cases of complications that could be directly attributed to lidocaine toxicity. Earlier studies have shown that doses of lidocaine well in excess of $5 \mathrm{mg} \cdot \mathrm{kg}^{-1}$ for bronchoscopy are tolerated by patients and rarely produce toxic blood levels $[11,14]$. However, in a report of 48,000 bronchoscopies by SuRATT et al. [15], there were six documented cases of convulsion, which were felt to be due to topical anaesthetic toxicity, although drug doses were not stated.

Use of antimuscarinics (29\%) prior to bronchoscopy was less common than in a previous British study (87\%) [5], but contrasts with a recent North American survey, which showed that more than twothirds of physicians still prescribed these agents [4]. There is no convincing evidence that antimuscarinic drugs provide any benefit to bronchoscopy.

Administration of antibiotic prophylaxis against endocarditis was common. Although endocarditis after bronchoscopy has been described [16], it would appear rare. Bronchoscopy is, however, a recognized cause of bacteraemia, and in view of this, antibiotic prophylaxis is probably justified.

Patient monitoring throughout the procedure was generally in accordance with established recommendations [2]. However, elecrocardiogram (ECG) monitoring was never undertaken by more than one-half of responders, which contrasts sharply with North American practice, where $85 \%$ of physicians routinely monitor the ECG during bronchoscopy [4]. Continuous ECG monitoring is recommended, as myocardial ischaemia during the procedure is common, particularly in the elderly [17].

Hypoxaemia during bronchoscopy results from a combination of sedation, topical anaesthesia, and mechanical obstruction from the endoscope. Supplemental oxygen can avoid hypoxaemia in most cases [18], and its routine use seems logical.

Low levels of clinical staffing during bronchoscopy (single assistant in 5\% responses) may prove inadequate in the event of an emergency, and the authors feel that at least two trained assistants should be present to aid specimen handling and ensure adequate patient monitoring throughout the procedure. Reported wearing of gloves during bronchoscopy was high, but routine use of other protective clothing as previously recommended [2], was low.

Differences in the sampling methods performed were noted, particularly when the bronchoscopic appearances were normal or showed VOT. When sampling from VOTs any additional yield from cytological samples will depend partly on the number of endobronchial biopsies taken. As a cost-effective means of diagnosis, some authors recommend obtaining cytological samples when occluding tumour is seen, but analysing them only if the biopsy sample is nondiagnostic. Use of cytological samples in the diagnosis of malignancy when 
bronchoscopic appearances are normal or show only airway oedema/compression, provide a greater input to the overall diagnostic yield. Cytology alone provided the only means of diagnosis via the bronchoscope in $34.2 \%$ and $34.5 \%$ of bronchoscopically nonvisible tumours in two series [19, 20], with washings and brushing giving complementary yields. Furthermore, the yield from wash and brush cytology specimens appears even greater when fluoroscopic guidance is used [21, 22].

Only a small number of physicians performed bronchial biopsies (blindly or with fluoroscopic guidance) in patients with a pulmonary mass lesion and normal bronchoscopic appearances. This is perhaps surprising since diagnostic rates of $\leqslant 69 \%$ for malignant lesions have been reported with biopsy under radiographical screening, in such patients $[23,24]$.

Controversy exists over the need for fluoroscopic guidance when performing TBB. The safety of TBB without fluoroscopy has been documented [25]. However, Simpson et al. [5] found a significant reduction in the reported incidence of all pneumothorax when TBB was performed with fluoroscopy, compared to TBB without fluoroscopy. The current study showed a significant reduction only in the frequency of pneumothorax requiring drain insertion, following TBB when fluoroscopy was used.

Reported use of TBNA was low. This may have been due partly to the cost of disposable needles, but is also likely to be attributable to previous experience of a low yield, as well as a lack of familiarity and training with the technique. Low rates of utilization of TBNA have been reported previously in North America [4, 26]. This is despite a number of series highlighting the sensitivity and safety of TBNA via the flexible bronchoscope in diagnosing and staging central and peripheral lung tumours, with and without radiographical control. Furthermore, HAPONIK et al. [27] reported a significantly increased yield from TBNA after endoscopists underwent a period of training in the technique, combined with improved preparation of cytology samples.

A small number of large airway stent insertions and other specialist procedures were reported. This is likely to be due to the low number of physicians regularly performing these therapeutic interventions, some of whom may not have replied. Nonetheless, the finding that $15 \%$ of physicians had recent experience of airway stenting via the flexible bronchoscope is higher than the corresponding figure from a recent survey of North American chest physicians where only $4.6 \%$ were found to stent patients [4]. Although the current questionnaire did not ask the type of stent inserted by physicians, in the UK uncovered stents appear to be used in greater numbers than covered stents, a practice which is unusual in Europe (Personal communication, Boston Scientific). Complication rates reported from airway stents were similar to those reported previously [28].

The mortality rate reported from bronchoscopy of $0.045 \%$ was similar to that of a previous British survey $(0.04 \%)$ [5], but somewhat higher than other multicentre studies $(0.01-0.02 \%)[6,15]$.
Problems inherent with surveys of this nature include dependance on physicians' reported answers, which may have relied on memory alone. Furthermore, in view of the anonymous nature of the questionnaire, replies could not be verified and some physicians may also have been reluctant to provide full information. The completed response rate of $60 \%$ compares favourably with previous bronchoscopy survey returns of $31 \%$ [15] and 53\% [5]. Furthermore, as it is likely that many nonresponders did not perform bronchoscopy, the authors feel that this work represents a reasonable assessment of current bronchoscopic practice in the UK.

Despite the diversity apparent in this study, bronchoscopy appears in the main, to be a safe procedure. Further prospective studies are required to establish optimal anaesthetic and sedation regimes, and to evaluate the most efficient and cost-effective combinations of sampling procedures for bronchoscopic investigation of patients with suspected lung tumours. Acknowledgements. The authors would like to
thank all the physicians who contributed to the
work by completing the survey questionnaires.

\section{References}

1. Ikeda S. The flexible bronchofiberscope. Keio J Med 1968; 17: 1-16.

2. Harrison BDW. Guidelines for care during bronchoscopy. Thorax 1993; 48: 584.

3. Neale G. Reducing risks in gastro-enterological practice. Gut 1998; 42: 139-142.

4. Colt HG, Prakash UBS, Offord KP. Bronchoscopy in North America. J Bronchol 2000; 7: 8-25.

5. Simpson FG, Arnold AG, Purvis A, Belfield PW, Muers MF, Cooke NJ. Postal survey of bronchoscopic practice by physicians in the United Kingdom. Thorax 1986; 41: 311-317.

6. Credle WF, Smiddy JF, Elliot RC. Complications of fibreoptic bronchoscopy. Am Rev Respir Dis 1976; 109: 67-72.

7. Hatton MQF, Allen MB, Vathenen AS, Mellor E, Cooke NJ. Does sedation help in fibreoptic bronchoscopy? BMJ 1994; 309: 1206-1207.

8. Putinati S, Ballerin L, Corbetta L, Trevisani L, Potena A. Patient satisfaction with conscious sedation for bronchoscopy. Chest 1999; 115: 1437-1440.

9. Grieg JH, Cooper SM, Kasimbazi HJN, Monie RDH, Fennerty AG, Watson B. Sedation for fibre optic bronchoscopy. Respir Med 1995; 89: 53-56.

10. Ben-Shlomo I, Abd-EI-Khalim H, Ezry J, Zohar S, Tverskoy M. Midazolam acts synergistically with fentanyl for induction of anaesthesia. $\mathrm{Br} J$ Anaesth 1990; 64: 45-47.

11. Efthimiou J, Higenbotham T, Holt D, Cochrane GM. Plasma concentrations of lignocaine during fibreoptic bronchoscopy. Thorax 1982; 37: 68-71.

12. Keane D, McNicholas WT. Comparison of nebulised and sprayed topical anaesthesia for fiberoptic bronchoscopy. Eur Respir J 1992; 5: 1123-1125. 
13. Miller WC, Awe R. Effect of nebulized lignocaine on reactive airways. Am Rev Respir Dis 1975; 3: 739-741.

14. Sutherland AD, Santamaria JD, Nana A. Patient comfort and plasma lignocaine concentrations during fibreoptic bronchoscopy. Anaesth Intensive Care 1985; 1344: 3704-374.

15. Suratt PM, Smiddy JF, Gruber B. Deaths and complications associated with fiberoptic bronchoscopy. Chest 1976; 69: 747-751.

16. Jurado RL, Klein S. Infective endocarditis associated with fiberoptic bronchoscopy in a patient with mitral valve prolapse. Clin Infect Dis 1998; 26: 769-770.

17. Davies L, Mister R, Spence DP, Calverley PM, Earis JE, Pearson MG. Cardiovascular consequences of fibreoptic bronchoscopy. Eur Respir J 1997; 10: 695698.

18. Dubrawsky C, Awe RJ, Jenkins DE. The effect of bronchofiberscope examination on oxygenation statue. Chest 1975; 67: 137-140.

19. Mak VHF, Johnston IDA, Hetzel MR, Grubb C. Value of washings and brushings at fibreoptic bronchoscopy in the diagnosis of lung cancer. Thorax 1991; 45: 373-376.

20. Chau CH, Yew WW, Wong PC, Lee J, Wong CF. Usefulness of collecting routine cytological specimens during fiberoptic bronchoscopy for endoscopically visible and nonvisible lung carcinoma. Chest 1997; 111: 522-523.

21. Chaudhary BA, Yoneda K, Burki NK. Fibreoptic bronchoscopy: comparison of procedures used in the diagnosis of lung cancer. $J$ Thorac Cardiovasc Surg 1978; 76: 33-37.

22. Lam WK, So SY, Hsu C, Yu DYC. Fibreoptic bronchoscopy in the diagnosis of bronchial cancer: comparison of washings, brushings and biopsies in central and peripheral tumours. Clin Oncol 1983; 9: $35-42$.

23. Wightman AJ, Douglas AC. Fluoroscopically controlled transbronchial biopsy of solitary peripheral pulmonary lesions using the fibreoptic bronchoscope. Clin Radiol 1978; 29: 621-624.

24. Cortese DA, McDougall JC. Biopsy and brushing of peripheral lung cancer with fluoroscopic guidance. Chest 1979; 75: 141-145.

25. Anders GT, Johnson JE, Bush BA, Matthews JI. Transbronchial biopsy without fluoroscopy; a sevenyear perspective. Chest 1998; 94: 557-560.

26. Haponik EF, Shure D. Underutilization of transbronchial needle aspiration: experience of current pulmonary fellows. Chest 1997; 112: 251-253.

27. Haponik EF, Cappellari JO, Chin R, et al. Education and experience improve transbronchial needle aspiration performance. Am J Respir Crit Care Med 1995; 151: $1998-2002$.

28. Monnier P, Mudry A, Stanzel F, et al. The use of the covered wallstent for the palliative treatment of inoperable tracheobronchial cancers: a prospective, multicentre study. Chest 1996; 110: 1161-1168. 